\title{
Characterizing vibration signals utilizing morphological pattern spectrum for gear fault diagnosis
}

\author{
*Bing Li, Min Gao, Qing-chen Guo, Fang Liu \\ Forth department, Mechanical Engineering College, No.97,He-ping West Road, Shi Jia-zhuang, 、 \\ He Bei province, PR China, 050003 \\ rommandy@163.com
}

Keywords: vibration signals; mathematical morphology; morphological pattern spectrum; gear fault diagnosis.

Abstract: In this investigation, we present an alternative technique to characterize the vibration signals by utilizing the morphological pattern spectrum (MPS) based on the mathematical morphology theory. Different from the conventional morphological pattern spectrum which is based on morphological open operator, we extend the morphological pattern spectrum with other morphological operators. Vibration signals measured from a gearbox with three operating states are employed to evaluate the effectiveness of MPS. Experimental results have demonstrated the extended MPS calculated based on morphological erosion operator to be very effective on characterizing vibration signals for gear fault diagnosis.

\section{Introduction}

Characterizing the vibration signals with appropriate features is the most significant issue to machine fault diagnosis. The features are required to contain necessary discriminative information for classifiers to achieve a better classification performance in identifying mechanical faults.

In this work, we present an alternative way to characterize the vibration signals for gear fault diagnosis based on the morphological pattern spectrum. The morphological pattern spectrum (MPS), which can be computed using a technique from mathematical morphology known as granulometries, has been broadly used in image analysis area [1-3]. We experimentally evaluate and compare the MPS technique with the traditional feature extraction methods. Experimental results have ascertained the effectiveness of the MPS technique for accurate fault diagnosis of gear.

\section{Morphological pattern spectrum}

Basic theories on mathematical morphology. The basic operators of MM include dilation, erosion, opening and closing. The definitions of these morphological operators are given as follow.

Let $f(n)$ be the discrete one dimension signal over a domain $F=\{0,1,2, \cdots, N-1\}$ and let $g(m)$ be the structure element over a domain $G=\{0,1,2, \cdots M-1\}$, the dilation and erosion operators are defined as:

$$
\begin{aligned}
& (f \oplus g)(n)=\max _{m \in G}\{f(n-m)+g(m)\} \\
& (f \Theta g)(n)=\min _{m \in G}\{f(n+m)-g(m)\}
\end{aligned}
$$

where $\oplus$ represents the operator of dilation and $\Theta$ represents the operator of erosion. More details about the morphological operations and the structure elements can be found in ${ }^{[1]}$.

The opening and closing operators are constructed based on the dilation and erosion operators, which are defined as:

$$
\begin{aligned}
& (f \circ g)(n)=(f \Theta g \oplus g)(n) \\
& (f \bullet g)(n)=(f \oplus g \Theta g)(n)
\end{aligned}
$$


Where $\circ$ means the opening operator and $\bullet$ means the closing operator. In practice, the operators are chosen according to the specific signal type.

Morphological pattern spectrum. The concept of morphological pattern spectrum or granulometric curve was introduced first by Matheron [2] as tools to extract size distributions from binary images. A measure $U(X)$ can be defined to quantify the rate at which $X$ is being sieved. In most cases, $U(X)$ is simple taken to be the area of $X$. Then the morphological pattern spectrum (MPS) [3] can be defined as:

$$
P S_{X}(r)=-\frac{d U(X \circ r g)}{d r}
$$

where $r$ is the size scale, $g$ is the unit structure element and $r g$ is defined as:

$$
r g=\underbrace{g \oplus g \oplus \cdots \oplus g}_{r-1 \text { times }}
$$

Furthermore, the MPS can be extended to one-dimensional signals. In this case, the measure $U(X)$ can be defined as the integral (sum of the gray levels) of $X$ over the signal domain, means the area of $X$. In the phase of our study, the vibration signals from different gear states can be characterized by the morphological pattern spectrum. By classifying the vibration signals using the MPS more accurately, we can obtain a better performance for gear fault diagnosis.

It can be observed in Eq.(5) that, the conventional MPS is calculated based on the morphological opening operator, which has been proved to be very effective in analyzing binary images. However, it may not be in analyzing one-dimensional signals. So we extend the MPS to be calculated by four types of morphological operators, mean the morphological dilation, morphological erosion, and morphological open and morphological close, in our work.

\section{Experimental results}

Gear experiment system. The vibration data of gear used in this paper was acquired from a two stage gearbox. Two different type fault types, i.e. gear root crack and tooth wear were set to middle gear B in the experiment. Therefore, three gear states i.e., normal condition, middle gear B tooth crack and middle gear tooth $\mathrm{B}$ wear were simulated in the test for evaluating the proposed methods.

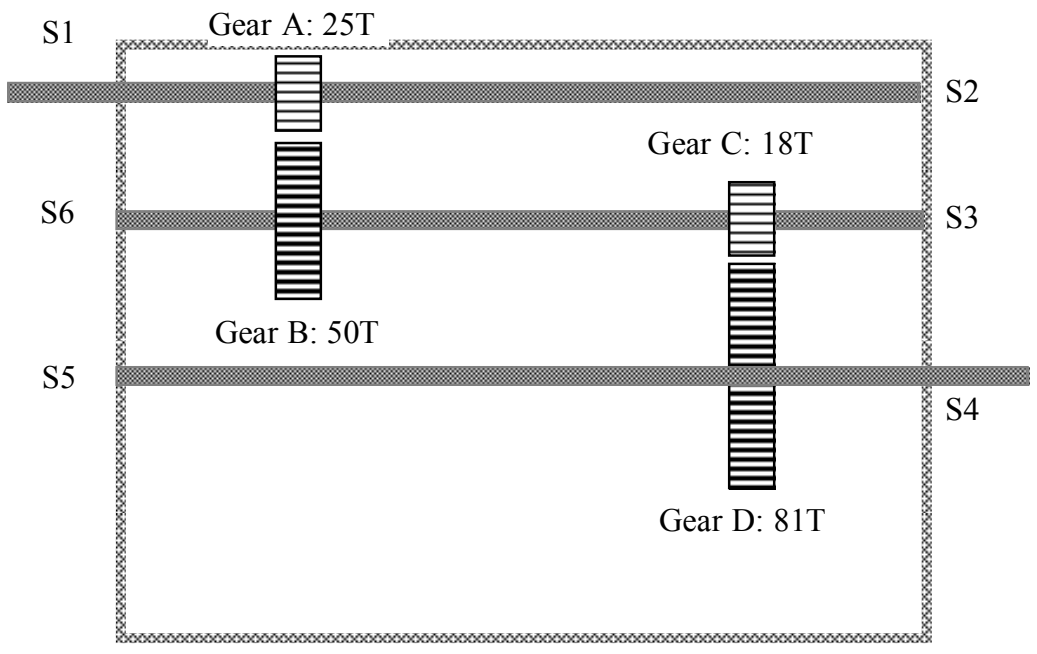

Figure 1 Structure of the experimental two-stage gearbox

Six accelerator sensors, as shown in Fig.1, were attached on the top of the six bearing bases to pick up the vibration signals. The bandpass of the sensors is $0 \sim 20 \mathrm{~K} \mathrm{~Hz}$. The rotation speed of the input shaft was set to be $1500 \mathrm{rpm}$ and the load was set be $100 \mathrm{NM}$ in the experiment. The sample frequency is 
6400 and the sample number is 6400 points. In this work, the signals acquired from the S6 were analyzed due to it is the nearest sensor to middle gear B. We collected 40 samples for each gear state and there 120 samples were collected in total. Fig. 2 has demonstrated the waveform of vibration signal of three gear states acquired from S6.
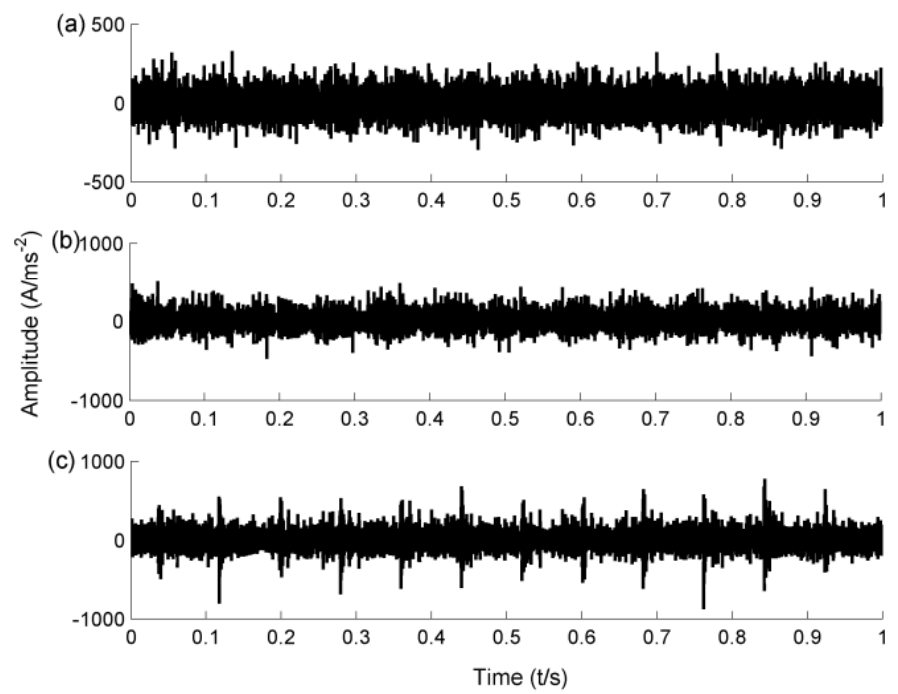

Figure 2 Vibration signals from gear with three states:

(a) normal; (b) middle gear B tooth crack; (c) middle gear B tooth wear

Characterizing vibration signals using Morphological pattern spectrum (MPS). In this subsection, the MPS is employed to characterize the vibration signals. In this work, we utilize the four morphological operators, mean the dilation, erosion, open and close to calculate the morphological pattern spectrum. The flat structure element is utilized in our work. The scale $r$ is set to be $1 \sim 30$.
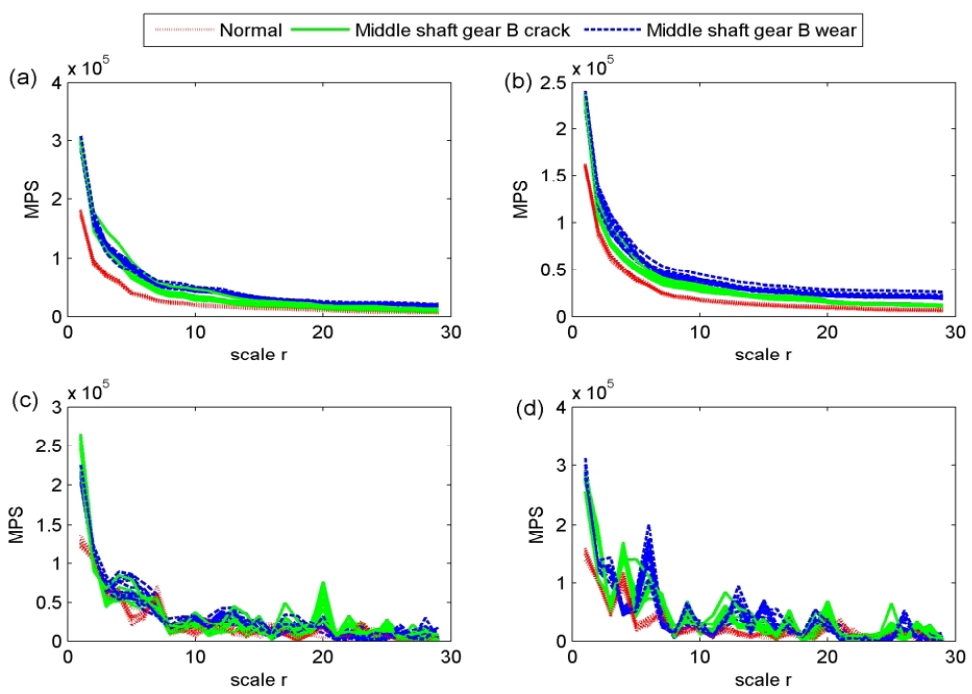

Figure 3 The morphological pattern spectrum of three gear states calculated by:

(a) morphological dilation; (b) morphological erosion; (c) morphological open; (d) morphological close

Fig. 3 illustrates the MPS calculated by four types of morphological operators, respectively. In each figure, the MPS of five samples of each gear state are displayed. It can be observed that, the three gear states can be discriminated by the MPS. We will assess the performances of the above four MPSs for classifying the gear states in next subsection.

Classification results. In order to evaluate the performances of the above feature extraction approaches, three popular classifiers means the $K$ nearest neighbor classifier (KNNC) [4] , Naïve Bayes classifier) (NBC) [5] and least-square support vector machine (LS-SVM) [6] were employed as 
induction algorithms for gear fault classification. The KNNC and NBC were implemented by utilizing the Matlab Toolbox for Pattern Recognition (PRTools 4.1) [7]. The LS-SVM was implemented by the LS-SVMlab1.5, which can be downloaded from [8]. The parameters of the LS-SVM were optimized by cross-validation method.

As mentioned in section 3.1, we collected 120 samples from S6 in the experiment. The dataset was segmented to two parts, 60 samples (20 samples of each gear state) were employed as the training dataset and the other 60 samples were used as testing dataset. We did this segmentation randomly for 20 times for each dataset to get a more robust evaluation results. The classification results of the traditional statistical parameters (maximum, minimum, peak-peak, RMS, kurtosis, skewness, crest factor, impulse factor, shape factor and clearance factor) and the four MPSs using KNNC, NBC and SVM are summarized in Table 1.

Table 1 Classification rates of three classifiers based on statistical parameters and four type MPSs for gear fault diagnosis

\begin{tabular}{cccc}
\hline \multirow{2}{*}{ Feature subsets } & \multicolumn{3}{c}{ Classification accuracies (\%) } \\
\cline { 2 - 4 } Statistical parameters & KNNC & NBC & SVM \\
\hline MPS Dilation & 86.44 & 82.44 & 89.33 \\
MPS Erosion & 95.33 & 89.78 & 96.44 \\
MPS Open & 99.11 & 96.44 & 100 \\
MPS Close & 96.67 & 92.44 & 98.56 \\
\hline
\end{tabular}

It can be noticed in Table 1 that, the MPSs with all the four type morphological operators show constant better performances over the statistical features. Another finding is that MPS calculated by morphological erosion gives the best classification results constantly on the three classifiers. It accords with the visual inspection in Figure 3. Therefore, the MPS calculated by morphological erosion seems to be an attractive scheme to characterize vibration signals for accurate classification of gear faults in applications.

\section{Conclusion}

This investigation has proposed an available feature extraction scheme by utilizing the morphological pattern spectrum (MPS) for gear fault diagnosis. Vibration signals acquired from a gearbox with three operating states were used to evaluate the effectiveness of the morphological pattern spectrum. Experimental results on classifying the three gear operating states have revealed the superiority of the proposed MPS over the traditional statistical feature extraction methods.

Furthermore, we investigated the effects of the morphological operators, mean dilation, erosion, open and close on the discriminative capacity of gear defects. Results have demonstrated that the morphological erosion achieves the best performance on identifying the gear operating states. Our study has demonstrated that the morphological pattern spectrum to be a desirable technique to characterize the vibration signals for gear fault diagnosis. It can be also employed for faults identification on other mechanical equipments such as bearings and engines.

\section{Acknowledgments}

This study was supported by the National Natural Science Foundation of China, under project number 51205405 . 


\section{References}

[1] Maragos, P.,R.W. Schafer. Morphological Filters - Part I: Their Set - Theoretic Analysis And Relations To Linear Shift - Invariant Filters. IEEE Transactions on Acoustics, Speech, and Signal Processing, 1987, ASSP-35(8):1153-1169.

[2] Matheron, G., Random sets and integral geometry. 1975, New York: Wiley.

[3] Maragos, P. Pattern spectrum and multiscale shape representation. IEEE Transactions on Pattern Analysis and Machine Intelligence, 1989, 11(7):701-716.

[4] Grother, P.J., G.T. Candela,J.L. Blue. Fast implementations of nearest neighbor classifiers. Pattern Recognition, 1997, 30(3):459-465.

[5] Yager, R.R. An extension of the naive Bayesian classifier. Information Sciences, 2006, 176(5):577-588.

[6] Suykens, J.A.K.,J. Vandewalle. Least squares support vector machine classifiers. Neural Processing Letters, 1999, 9(3):293-300.

[7] R.P.W. Duin, P.J., P. Paclik, E. Pekalska, D. de Ridder, D.M.J. Tax, S. Verzakov. PRTools4.1, A Matlab Toolbox for Pattern Recognition. Delft University of Technology, 2007.

[8] Wu, J.D., M.R. Bai, F.C. Su,C.W. Huang. An expert system for the diagnosis of faults in rotating machinery using adaptive order-tracking algorithm. Expert Systems With Applications, 2009, 36(3 PART 1):5424-5431. 